\title{
Extravasation Wound Care in the Neonatal Intensive Care Unit
}

\author{
Kyu Sang Cho' , Hee Chang Ahn¹, Jang Hyun Lee², Soo Yeon Lim, Hyun Ju Lee³, Hyun Kyung Park ${ }^{3}$ \\ ${ }^{1}$ Department of Plastic and Reconstructive Surgery, Hanyang University College of Medicine, Seoul; ${ }^{2}$ Department of Plastic and Reconstructive Surgery, \\ Hanyang University Guri Hospital, Hanyang University College of Medicine, Seoul; ${ }^{3}$ Department of Pediatrics, Hanyang University College of Medicine, Seoul, \\ Korea
}

Abstract

\begin{abstract}
Background: Extravasation injuries occurring in neonates can have severe consequences. Because many clinicians are not familiar with the vesicant-like effect of several commonly used medications, early extravasation wound management can be difficult. We aimed to share our experience with irritant-related and vesicant-related injuries leading to neonatal extravasation wounds.

Methods: This retrospective study included 36 premature neonatal patients with extravasation wounds. Patients were classified into four groups based on extravascular staging and clinical wound conditions. We compared groups according to the following factors: sex, birth weight, prematurity, wound healing duration, days since the intravenous line was placed, wound severity, use of antibiotics, complications, treatment, type of intravenous fluid, and intravenous cannulation sites.

Results: Neonatal birth weight was proportional to wound severity. Except in cases involving necrosis wounds, the lesion size increased with severity. Extravasation events occurred an average of 3.2 days after cannulation. Neonatal parenteral nutrition formulas and antibiotics acted as vesicants.

Conclusion: Intravenous cannulation sites must be closely monitored in neonates, particularly during the first 3 days after cannulation. Monitoring is particularly important for larger neonates because intravenous fluid is administered based on body weight. Detailed knowledge about the vesicant fluid is required.
\end{abstract}

Keywords: Irritants; Re-epithelialization; Newborn; Wound healing; Wound infection

\section{Introduction}

The intravenous approach is important for the administration of fluids and medications used to manage critically ill neonates. Successful placement of an intravenous cannula in a neonate requires skill [1]. However, regardless of the skill of healthcare providers, intravenous cannulation in neonates can be complicated by extravasation events. In many studies, the prevalence of at least one extravasation event in neonates was as high as $70 \%$ [2-4]. This high prevalence was attributable to the immature vasculature of premature infants, which makes the course of blood vessels less predictable and more vulnerable to damage compared with the blood vessels of adults [5]. During and after cannulation, the site does not remain fixed, which makes neonates vulnerable to extravasation.

Neonates are at increased risk for infection because of their immature tissues and thin vessel layers [6]. Furthermore, several substances such as vesicant fluids used in parenteral therapy can be harmful to body tissues and cause damage such as blistering or necrosis when extravasation occurs [7,8]. Moreover, large volumes of intravenous fluid administered in relation to body volume might result in extravasation events.

Many intravenous fluids used for neonates can be categorized as either vesicants or

\section{Original Article}

Received: November 1, 2018

Revised: January 29, 2019

Accepted: February 1, 2019

\section{Corresponding author: Hee Chang Ahn, M.D., Ph.D.}

Department of Plastic and Reconstructive Surgery, Hanyang University College of Medicine, 222-1 Wangsimni-ro, Seongdonggu, Seoul 04763, Korea

Tel: +82-2-2290-8560

Fax: +82-2-2295-7671

E-mail: ahnhc@hanyang.ac.kr

This is an Open Access article distributed under the terms of the Creative Commons Attribution Non-Commercial License (http://creativecommons.org/licenses/by-nc/4.0/) which permits unrestricted non-commercial use, distribution, and reproduction in any medium, provided the original work is properly cited.

(C) 2019 Korean Wound Management Society 
irritants. For example, total parenteral nutrition fluids and many kinds of antibiotics act as vesicants [9-12]. Treatment of extravasation injuries sustained by neonates remains highly problematic in many countries. However, evidence regarding the most appropriate protocol to minimize damage due to extravasation events is insufficient. Only a few studies have suggested wound manage procedures [8,13-17].

Extravasation events involve severe complications such as the progression of a relatively mild wound (tissue edema, redness, or vasculitis) to skin necrosis or an abscess [18]. Despite the high prevalence of extravasation events and their severity, it seems that many medical teams do not know which intravenous fluid leads to severe consequences, which requires early intervention, and which treatment should be administered. We aimed to share our experience with irritant-related and vesicant-related injuries leading to neonatal extravasation wounds.

\section{Methods}

Thirty-six patients with extravasation events who were referred to the plastic and reconstructive surgery department from the neonatal intensive care unit between January 2012 and June 2017 were evaluated during this retrospective study. The study was approved by the Institutional Review Board of Hanyang University Hospital (IRB No. 2017-11-034) and performed in accordance with the principles of the Declaration of Helsinki. The informed consent was waived.

The following parameters were analyzed: sex, birth weight, days since the intravenous line was placed, type of intravenous fluid, wound severity, treatment, wound healing duration, use of systemic antibiotics, complications, and intravenous cannulation site. Wound severity was determined in the Plastic and Reconstructive Surgery department after the patients were referred from the neonatal intensive care unit (ICU). This may have resulted in a delay of 1 to 3 days after extravasation.
Total parenteral nutrition fluid, which included lipid emulsion (Intralipid, Fresenius Kabi, Austria), was involved in 20 of the extravasation patients. Antibiotic extravasation accidents occurred with ampicillin (3 cases), gentamicin sulfate plus ampicillin ( 2 cases), and piperacillin/tazobactam ( 2 cases). Total parenteral nutrition and antibiotics are classified as vesicants in neonates.

To stage extravasation wounds, we referred to a study by the Infusion Nurses Society [19]. However, patients with extravasation wounds were usually referred to the plastic surgery department 2 to 3 days after the extravasation event occurred. Therefore, it was difficult to conventionally classify the wounds. Because of this limitation, we categorized wounds from a clinical point of view.

Patients were categorized into four groups based on the nature of the wounds when they were referred to the Plastic and Reconstructive Surgery department. These categories included color change (level I), swelling and/or bulla formation (level II), skin abrasion and/or site infection (level III), and necrosis (level IV). Patients in level I and level II categories were managed using conservative dressings. Patients with a more clinically severe status (level III or level IV) were provided interventions such as hyaluronidase injection, incision, drainage, and debridement of necrotic materials (Table 1). Millimeter grid paper was used to measure the surface area of wounds with complex shapes. Interventions included observation after dressing, hyaluronidase injection, aspiration or incision of bullae or abscess, and incision and debridement of necrotic materials.

A statistical analysis was performed using the SAS program version 9.4 (SAS Institute, Cary, NC, USA). Other analyses were performed using the chi-square test. Fisher exact test was also used when the sample size was insufficient to perform a chi-square test. The Wilcoxon rank-sum test, Kruskal-Wallis test, and Jonckheere-Terpstra one-sided test were also used.

Table 1. Extravasation wound classifications

\begin{tabular}{|c|c|c|c|c|}
\hline & Grade 1 & Grade 2 & Grade 3 & Grade 4 \\
\hline \multirow[t]{5}{*}{ Traditional classifications [19] } & Pain at infusion site & Pain at infusion site & Pain at infusion site & Pain at infusion site \\
\hline & & Swelling & Swelling & Swelling \\
\hline & & No skin blanching & Skin blanching & Skin blanching \\
\hline & & & Cool blanched area & Cool blanched area \\
\hline & & & & Reduced capillary refill \\
\hline \multirow[t]{2}{*}{ Clinical observation } & Level 1 & Level 2 & Level 3 & Level 4 \\
\hline & Color change & Bulla & Cellulitis, abscess & Necrosis \\
\hline
\end{tabular}




\section{Results}

Thirty-six neonates were included in this study. There was an equal distribution of the sexes. The mean birth weight was $1,836 \mathrm{~g}$ (standard deviation [SD], $\pm 1,015$ ). The mean body weight at admission was $2,166.4 \mathrm{~g}$. The mean age at admission was 58.2 days. The average wound healing duration was 18.7 days. The mean time from intravenous cannulation to wound occurrence was 3.2 days. The mean wound size was $224.7 \mathrm{~cm}^{2}$ (Table 2).

Fourteen cases (38.9\%) were classified as level I, eight cases (22.2\%) were classified as level II, seven cases (19.4\%) were classified as level III, and seven cases (19.4\%) were classified as level IV.

The most common intravenous cannulation site was the distal upper limb (50.0\%), followed by the distal lower limb (22.2\%), proximal upper limb (19.4\%), and proximal lower

Table 2. Patients demographics $1(n=36)$

\begin{tabular}{|c|c|}
\hline Characteristic & Value \\
\hline Male:female & $18: 18$ \\
\hline Birth weight (g) & $1,836 \pm 1,015$ \\
\hline Body weight at admission (g) & $2,166.4 \pm 1,619.1$ \\
\hline Age at admission (day) & $58.2 \pm 141.2$ \\
\hline Wound healing duration (day) ${ }^{\text {a) }}$ & $18.7 \pm 20.6$ \\
\hline Lesion size $\left(\mathrm{mm}^{2}, \mathrm{n}=16\right)$ & $224.7 \pm 172.0$ \\
\hline Cannulation duration (day) $)^{\text {b) }}$ & $3.2 \pm 4.2$ \\
\hline
\end{tabular}

Values are presented as number or mean \pm SD.

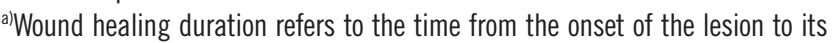
cure; ${ }^{b}$ Cannulation duration means the duration of intravenous fluid infusion before lesion development. limb (5.6\%). Intravenous cannulation was performed on the scalp (2.8\%) for one patient.

Total parenteral nutrition was the most common substance involved in extravasation events (20 patients, $59.0 \%$ ), followed

Table 3. Patients demographics $2(n=36)$

\begin{tabular}{lc}
\hline Characteristic & No. of patients (\%) \\
\hline Location & $18(50.0)$ \\
Distal upper limb & $8(22.2)$ \\
Distal lower limb & $7(19.4)$ \\
Proximal upper limb & $2(5.6)$ \\
Proximal lower limb & $1(2.8)$ \\
Scalp & \\
Extravasated substance ( $=34)$ & $20(59.0)$ \\
Total parenteral nutrition (Intralipid) & $7(20.0)$ \\
Antibiotics & \\
Iron hydroxy-polymaltose complex & $4(12.0)$ \\
5\% Dextrose water & $3(9.0)$ \\
Wound severity & \\
Level I (induration) & $14(38.9)$ \\
Level II (bulla formation) & $8(22.2)$ \\
Level III (abscess) & $7(19.4)$ \\
Level IV (necrosis) & $7(19.4)$ \\
Intervention & $25(69.4)$ \\
Dressing only & $5(13.9)$ \\
Hyaluronidase injection & $6(16.7)$ \\
Incision only/l\&D intervention &
\end{tabular}

$I \& D$, incision and drainage.

${ }^{a)}$ Number of patients extravasated antibiotics: ampicillin (3 cases), gentamicin sulfate+ampicillin (2 cases), piperacillin/tazobactam, vancomycin (2 cases).

Table 4. Factors related to wound severity $(n=36)$

\begin{tabular}{|c|c|c|c|c|c|}
\hline \multirow{2}{*}{ Factor } & \multicolumn{4}{|c|}{ Wound severity } & \multirow{2}{*}{ P-value } \\
\hline & Levell & Level II & Level III & Level IV & \\
\hline Birth weight (g) & $1,525.5 \pm 764.4$ & $1,667.5 \pm 1,214.5$ & $1,835.7 \pm 902.3$ & $2,718.6 \pm 1,003.6$ & $<0.05$ \\
\hline Wound healing duration (day) & $9.8(3-38)$ & $26.3(2-80)$ & $17.0(3-35)$ & $29.7(3-86)$ & $<0.05$ \\
\hline Lesion size $\left(\mathrm{mm}^{2}, \mathrm{n}=16\right)$ & $267.5(120-400)$ & $320.0(160-600)$ & $400.0(400-400)$ & $87.5(25-225)$ & $<0.05$ \\
\hline Intervention & & & & & $<0.05$ \\
\hline Dressing only $(n=25)$ & $12(48.0)$ & $5(20.0)$ & $1(4.0)$ & $7(28.0)$ & \\
\hline Hyaluronidase injection $(n=5)$ & $3(60.0)$ & $1(20.0)$ & $1(20.0)$ & 0 & \\
\hline Incision/split incision/I\&D intervention $(n=6)$ & 0 & $1(16.7)$ & $5(83.3)$ & 0 & \\
\hline
\end{tabular}

Values are presented as mean \pm SD, median (range), or number (\%).

$I \& D$, incision and drainage.

a) Jonckheere-Terpstra one-sided test. 
by antibiotics (7 patients, 20.0\%), iron hydroxyl-polymaltose complex (4 patients, $12.0 \%$ ), and 5\% dextrose (3 patients, 9.0\%). The extravasated substance for two neonates was unknown because of insufficient charting (Table 3 ).

Table 4 shows factors related to wound severity. Neonatal birth weights were proportional to wound severity, and the relationship was statistically significant $(\mathrm{P}<0.05)$. Wound healing duration was proportional to wound severity. In the level I group, the mean duration was 9.8 days; in the level IV group, the mean duration was 29.7 days.

Lesion size increased gradually with the increasing level of wound severity, with the exception of level IV. The mean sizes were $267.5,320.0$, and $400.0 \mathrm{~mm}^{2}$ for levels I, II, and III, respectively. In contrast, the mean lesion size for level IV was much smaller than those of the other levels $\left(87.5 \mathrm{~mm}^{2}\right)$.

Twenty-five patients were placed under observation, and their wounds were dressed until they healed. For five patients, hyaluronidase was injected immediately after extravasation was noted. Some wounds progressed to a state of necrosis; subsequently, six patients required surgical intervention for rapid healing of the wound site (including incision and drainage, debridement, and coverage with a local flap).

Eleven of the 36 patients underwent hyaluronidase injection or surgical intervention. These interventions prevented the wounds from progressing to a state of necrosis and significantly decreased wound severity $(\mathrm{P}<0.05)$.

\section{Discussion}

This study found that total parenteral nutrition and antibiotics were the most commonly implicated substances in extravasation events. Wound severity was higher for heavier neonates. Lesion sizes were larger for more severe wounds, except in cases of necrosis. Therefore, heavier neonates may be more vulnerable to more severe wounds because intravenous dosages are determined based on body weight.

Extravasation of nonvesicant drugs usually does not cause tissue damage. In contrast, extravasation of vesicant agents can cause severe tissue damage. Infusion of vesicants, such as total parenteral nutrition, low $\mathrm{pH}$ molecules, and hyperosmolar fluid, and some vesicant-acting antibiotics should be performed carefully. Injuries may occur due to hyperosmotic solutions that lead to compartment syndrome, concentrated electrolyte solutions that possibly prolong muscle depolarization and lead to ischemia, drugs that alter intracellular $\mathrm{pH}$, and drugs that induce severe vasoconstriction and ischemia
[20].

Extravasation events are difficult to manage and can have a negative impact on neonatal patients. Therefore, early identification and treatment of such events are critical. Moreover, it is often difficult to predict the outcomes of extravasation events because they can be benign or severe.

Several previous studies have highlighted extravasation injuries, with up to $70 \%$ of injuries occurring in the upper limbs. In this study, we found a similar occurrence. The tendency of upper limb involvement likely reflects the easier access of intravenous fluid to the upper limb compared to the lower limb $[4,21]$.

In this study, wound severity was also related to the duration of wound management. The greater the wound severity, the longer the time required for treatment. In addition, severe wounds tended to be larger than less severe wounds; in contrast, necrotized wounds were usually small. Necrosis requires demarcation, which ultimately makes wounds appear smaller. However, as previous data demonstrated, necrotized wounds become deeper and require longer healing time than other types of wounds [13].

The mean time span between intravenous cannulation and extravasation events was 3.2 days (SD, 4.15 days), suggesting that cannulation sites should be carefully observed for at least this length of time (maximum, 7.35 days). This observation period might be sufficient to prevent neonatal extravasation wounds from forming.

Consistent with a previous study, the group in our study whose extravasated substance was total parenteral nutrition had a higher tendency for wounds that progressed in severity [1]. In our study, the group whose extravasated substance was iron hydroxyl-polymaltose complex tended to have less severe wounds compared to those of other groups.

This study included cases in which 5\% dextrose water and iron hydroxyl-polymaltose caused extravasation injuries. Extravasation wounds due to iron-containing substances are rare. Rather than skin necrosis, pigmentation is usually associated with iron preparations [22].

Clinicians usually manage extravasation wounds in one of four ways: observation and dressing (conservative treatment); hyaluronidase injection; aspiration; and incision [2,23]. In our study, patients in the level IV group were initially managed with conservative dressings. This could explain the delayed diagnosis and intervention that ultimately allowed the wound to progress to a state of necrosis in this group. In the level II and level III groups, surgical intervention was relatively common. 
According to a previous study, surgical interventions such as debridement and coverage with a local flap or incision and drainage might help stop wound progression [4].

Vesicant extravasation incidents are increasing. Non-antineoplastic vesicants are problematic for neonates; however, hyaluronidase has an important role as an effective counter to these agents. Subcutaneous hyaluronidase injections can be used to attempt to break down connective tissue and facilitate the absorption of the extravasated fluid into the vascular and lymphatic systems. Some researchers recommended that the administration of these injections should occur within 1 hour of extravasation, and that they will decrease the severity of tissue damage caused by extravasations [24]. However, infected or inflamed lesions, extravasation of vasoconstrictive drugs, and hypersensitive conditions are contraindications for hyaluronidase injections.

Wound site infection is mostly caused by the Staphylococcus species. In the group of patients who were not administered systemic antibiotics. There was a higher tendency for the wounds to progress to level III compared to patients in which antibiotics were administered. None of the neonates who were administered early systemic antibiotics developed a level III wound. Therefore, systemic antibiotics on the day of injury appeared to prevent wound infections. However, aggressive use of antibiotics could lead to other complications such as antibiotic-associated diarrhea or antibiotic-resistant infections. Therefore, antibiotic injections for infection control of extravasated wounds must be carefully re-evaluated using an evidence-based method. Prevention and early management are extremely important in minimizing the harm done by neonatal extravasation events. Medical teams managing neonates should be aware of all irritants and vesicant agents. Total parenteral nutrition formulas and many antibiotics act as vesicant agents in neonates; therefore, the first intravenous site should be closely observed for at least 48 hours. Early management, such as cold massage, saline irrigation, hyaluronidase injection, infection prevention, necrotic tissue debridement, and incision and drainage procedures, should be performed.

This study had some limitations. The sample size was relatively small; therefore, it was difficult to determine relationships between extravasation substances, doses, and wound severity based on our results. Furthermore, by the time patients were referred to the plastic and reconstructive surgery department, wounds had already progressed and changed. Therefore, early wound evaluation was not possible. Additionally, early management according to the extravasated agents was insuffi- cient. Because this study was retrospective, photographs of iron hydroxyl-polymaltose extravasation were not obtained. Moreover, thermal therapy has been proven to be beneficial in the injured area [25]. However, in this study, we only performed hyaluronidase injection, incision and drainage, or debridement and coverage with a local flap as treatment.

In conclusion, the medical team must be aware that many intravenous drugs act as irritants or vesicants in neonates. Therefore, preventive measures for vesicant extravasation must be performed along with careful infusions. If an extravasation is identified, then early intervention should be provided. Additionally, medical professionals must be aware of all well-known irritants and vesicants that may cause extravasation and their side effects.

\section{Conflict of interest}

No potential conflicts of interest relevant to this article are reported.

\section{Acknowledgments}

We thank Professor Yong Gyu Park of Catholic University, Seoul, Korea, for his contribution to the statistical analysis.

\section{References}

1. Wilkins CE, Emmerson AJ. Extravasation injuries on regional neonatal units. Arch Dis Child Fetal Neonatal Ed 2004;89: F274-5.

2. Reynolds BC. Neonatal extravasation injury: case report. Infant 2007;3:230-2.

3. Irving V. Managing extravasation injuries in preterm neonates. Nurs Times 2001;97:40-6.

4. Murphy AD, Gilmour RF, Coombs CJ. Extravasation injury in a paediatric population. ANZ J Surg 2017 Jul 6 [Epub]. https:// doi.org/10.1111/ans.14104.

5. Meszes A, Talosi G, Mader K, et al. Lesions requiring wound management in a central tertiary neonatal intensive care unit. World J Pediatr 2017;13:165-72.

6. Cartlidge PH, Fox PE, Rutter N. The scars of newborn intensive care. Early Hum Dev 1990;21:1-10.

7. Sauerland C, Engelking C, Wickham R, et al. Vesicant extravasation part I: mechanisms, pathogenesis, and nursing care to reduce risk. Oncol Nurs Forum 2006;33:1134-41.

8. Wickham R, Engelking C, Sauerland C, et al. Vesicant ex- 
travasation part II: evidence-based management and continuing controversies. Oncol Nurs Forum 2006;33:1143-50.

9. Aribit F, Laville J, Baron J. Extravasation injuries and subcutaneous aspiration in children. Rev Chir Orthop Reparatrice Appar Mot 2000;86:87-8.

10. Rosales CM, Jackson MA, Zwick D. Malassezia furfur meningitis associated with total parenteral nutrition subdural effusion. Pediatr Dev Pathol 2004;7:86-90.

11. Mohr LD, Reyna R, Amaya R. Neonatal case studies using active leptospermum honey. J Wound Ostomy Continence Nurs 2014;41:213-8.

12. Tobin C. Managing an extravasation wound in a premature infant. Wounds UK 2007;3:90-1.

13. Sardesai SR, Kornacka MK, Walas W, et al. latrogenic skin injury in the neonatal intensive care unit. J Matern Fetal Neonatal Med 2011;24:197-203.

14. Franck LS, Hummel D, Connell K, et al. The safety and efficacy of peripheral intravenous catheters in ill neonates. Neonatal Netw 2001;20:33-8.

15. Cho KY, Lee SJ, Burm JS, et al. Successful combined treatment with total parenteral nutrition fluid extravasation injuries in preterm infants. J Korean Med Sci 2007;22:588-94.

16. Casanova D, Bardot J, Magalon G. Emergency treatment of accidental infusion leakage in the newborn: report of 14 cases. Br J Plast Surg 2001;54:396-9.

17. Rose V, Turner M, Harris PA, et al. Emergency treatment of accidental infusion leakage in the newborn. Br J Plast Surg 2002;55:89.

18. Lee TG, Chung S, Chung YK. A retrospective review of iatrogenic skin and soft tissue injuries. Arch Plast Surg 2012;39: 412-6.

19. Infusion Nurses Society. Infusion nursing standards of practice. J Infus Nurs 2006;29(1 Suppl):S1-92.

20. Goutos I, Cogswell LK, Giele H. Extravasation injuries: a review. J Hand Surg Eur Vol 2014;39:808-18.

21. Ghanem AM, Mansour A, Exton R, et al. Childhood extravasation injuries: improved outcome following the introduction of hospital-wide guidelines. J Plast Reconstr Aesthet Surg 2015;68:505-18.

22. Hermitte-Gandoliere A, Petitpain N, Lepelley M, et al. Cutaneous pigmentation related to intravenous iron extravasation: analysis from the French pharmacovigilance database. Therapie 2018;73:193-8.

23. Harris PA, Bradley S, Moss AL. Limiting the damage of iatrogenic extravasation injury in neonates. Plast Reconstr Surg 2001;107:893-4.

24. Bellin MF, Jakobsen JA, Tomassin I, et al. Contrast medium extravasation injury: guidelines for prevention and management. Eur Radiol 2002;12:2807-12.

25. Firat C, Erbatur S, Aytekin AH. Management of extravasation injuries: a retrospective study. J Plast Surg Hand Surg 2013; 47:60-5. 